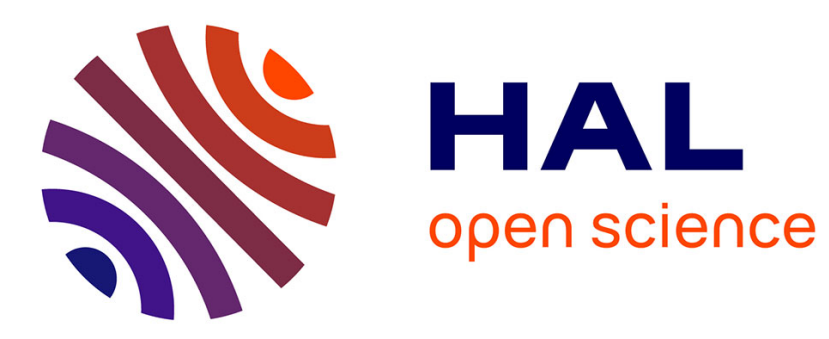

\title{
Generalized Inverted Kumaraswamy Generated Family of Distributions: Theory and Applications
}

Farrukh Jamal, M Elgarhy, M Arslan Nasir, Gamze Ozel, Naushad Mamode Khan

\section{- To cite this version:}

Farrukh Jamal, M Elgarhy, M Arslan Nasir, Gamze Ozel, Naushad Mamode Khan. Generalized Inverted Kumaraswamy Generated Family of Distributions: Theory and Applications. 2018. hal01907258

\section{HAL Id: hal-01907258 \\ https://hal.science/hal-01907258}

Preprint submitted on 28 Oct 2018

HAL is a multi-disciplinary open access archive for the deposit and dissemination of scientific research documents, whether they are published or not. The documents may come from teaching and research institutions in France or abroad, or from public or private research centers.
L'archive ouverte pluridisciplinaire HAL, est destinée au dépôt et à la diffusion de documents scientifiques de niveau recherche, publiés ou non, émanant des établissements d'enseignement et de recherche français ou étrangers, des laboratoires publics ou privés. 


\title{
Generalized Inverted Kumaraswamy Generated Family of Distributions: Theory and Applications
}

\author{
a*Farrukh Jamal, ${ }^{b}$ M. Elgarhy, ${ }^{\mathrm{c}} \mathrm{M}$. Arslan Nasir, ${ }^{\mathrm{d}}$ Gamze Ozel and ${ }^{\mathrm{e}}$ Naushad Mamode Khan. \\ ${ }^{a}$ Department of Statistics, Government S.A. Post-Graduate College, \\ Dera Nawab Sahib, Pakistan. \\ ${ }^{b}$ Vice Presidency for Graduate Studies and Scientific Research, \\ Jeddah University. \\ ${ }^{c}$ Department of Statistics, Government Degree College, \\ Lodhran, Pakistan. \\ ${ }^{d}$ Department of Statistics, Hacettepe University, \\ Ankara, Turkey. \\ ${ }^{e}$ Department of Economics and Statistics, University of Mauritius, \\ Reduit, Mauritius. \\ *Email: drfarrukh1982@gmail.com
}

October 18, 2018

\begin{abstract}
This paper proposes a new generator function based on the inverted Kumaraswamy distribution "Generalized Inverted Kumaraswamy-G" family of distributions. We provide a comprehensive account of some of its mathematical properties that include the ordinary and incomplete moments, quantile and generating functions and order statistics. The infinite mixture representations for probability density and cumulative density and entropy functions of the new family are also established. The density function of the $i$ th order statistics is expressed as an infinite linear combination of baseline densities and model parameters are estimated by maximum likelihood method. Four special models of this family are also derived along with their respective hazard rate functions. The maximum likelihood estimation (MLE) method is used to obtain the model parameters. Monte Carlo simulation experiments are executed to assess the performance of the MLE estimators under the corresponding generated models while some data applications are also illustrated.
\end{abstract}

Keywords - Kumaraswamy distribution, quantile function, Simulation, maximum likelihood estimation.

Mathematics Subject Classification. 60E05, 62E15, 62F10.

\section{Introduction}

In the recent times, there has been an increased interest in applying some inverted distributions to data applications in the areas of medical, economic and engineering sciences (See Calabria and 
Pulcini (1990), Al-Dayian (1999),Abd El-Kader et al. (2003), Prakash (2012)). Such distributions include the inverse Weibull, inverted Burr Type XII, the Pareto Type I and the exponentiated inverted Weibull models. However, to further improve the goodness of fit especially in exploring tail properties, researchers have also considered to derive new generators for univariate continuous families of distributions by introducing one or more additional shape parameter(s) to the baseline distribution.

In this context, some well-known generators are the beta- $G$ by Eugene et al. (2002) and Jones (2004), Kumaraswamy-G (Kw-G) by Cordeiro and de Castro (2011), McDonald-G (Mc-G) by Alexander et al. (2012), gamma-G (type 1) by Zografos and Balakrishnan (2009), gamma-G (type 2) by Ristic and Balakrishnan (2012), gamma-G (type 3) by Torabi and Montazari (2012), logistic-G by Torabi and Montazari (2014), exponentiated generalized-G by Cordeiro et al. (2013), transformedtransformer (T-X) by Alzaatreh et al. (2013), Weibull-G by Bourguignon et al. (2014), Lomax$\mathrm{G}$ by Cordeiro et al. (2014b), and logistic-X by Tahir et al. (2014). Other recent family of distributions are Garhy-G by Elgarhy et al. (2016), Kumaraswamy Weibull-G by Hassan and Elgarhy (2016a), exponentiated Weibull-G by Hassan and Elgarhy (2016b), additive Weibull-G by Hassan and Hemeda (2016), exponentiated extended-G by Elgarhy et al. (2017), and type II half logistic-G by Hassan et al. (2017).

Most recently,

Al-Fattah et al. (2017) introduced the inverted Kumaraswamy (IKw) distribution with two positive shape parameters $\alpha>0$ and $\beta>0$, where the cumulative distribution function (cdf) is given by

$$
\Pi(x ; \alpha, \beta)=\left[1-(1+x)^{-\alpha}\right]^{\beta}, \quad x>0
$$

The probability density function (pdf) corresponding to (1.1) is

$$
\pi(x ; \alpha, \beta)=\alpha \beta(1+x)^{-\alpha-1}\left[1-(1+x)^{-\alpha}\right]^{\beta-1} .
$$

The curves of the pdf show that the IKw distribution exhibits a long right tail and as illustrated in the paper by Al-Fattah et al. (2017), IKw produces optimistic predictions of rare events occurring in the right tail of the distribution as compared with other distributions. However, in many applied areas such as lifetime analysis, finance and insurance, the data are seen to exhibit heavy-tail while in practice, there is also a strong need to generate distributions with symmetric, left-skewed, rightskewed and reverse J-shaped and hence to provide consistently better fits than other generated models under the same underlying distribution.

The basic motivations for obtaining GIKw-G family in practice are: (i) to make the kurtosis more flexible as compared to the baseline model; (ii) to produce skewness for symmetrical distributions; (iii) to construct heavy-tailed distributions that are not longer-tailed for modeling real data; (iv) to generate distributions with symmetric, left-skewed, right-skewed and reversed-J shape; (v) to provide consistently better fits than other generated models under the same underlying distribution.

In this context, this paper proposes a new generalized Inverted Kumaraswamy family of distributions (GIKw-G) using the generator $\frac{G^{\gamma}}{1-G^{\gamma}}$. For the conditions on the baseline distribution see Alzaatreh et al. (2013). 
The cdf of the new family, GIKw-G, is given by

$$
\begin{aligned}
F(y) & =\alpha \beta \int_{0}^{\frac{G^{\gamma}(x, \xi)}{1-G^{\gamma}(x, \xi)}}(1+y)^{-\alpha-1}\left[1-(1+y)^{-\alpha}\right]^{\beta-1} d y \\
& =\left[1-\left(1-G^{\gamma}(x, \xi)\right)^{\alpha}\right]^{\beta} .
\end{aligned}
$$

where the corresponding density function to (3) is given by

$$
f(x)=\alpha \beta \gamma g(x, \xi) G^{\gamma-1}(x, \xi)\left(1-G^{\gamma}(x, \xi)\right)^{\alpha-1}\left[1-\left(1-G^{\gamma}(x, \xi)\right)^{\alpha}\right]^{\beta-1}, \quad \alpha, \beta, \gamma>0 .
$$

where $\xi$ is the parametric space of the baseline distribution. Now onward $G(x, \xi)=G(x)$ and $g(x, \xi)=g(x)$. The survival, hazard rate and reversed hazard rate functions of the GIKw-G family are, respectively, given by

$$
\begin{gathered}
S(x)=1-\left[1-\left(1-G^{\gamma}(x)\right)^{\alpha}\right]^{\beta}, \\
h(x)=\frac{\alpha \beta \gamma g(x) G^{\gamma-1}(x)\left(1-G^{\gamma}(x)\right)^{\alpha-1}\left[1-\left(1-G^{\gamma}(x)\right)^{\alpha}\right]^{\beta-1}}{1-\left[1-\left(1-G^{\gamma}(x)\right)^{\alpha}\right]^{\beta}}, \\
H(x)=\frac{\alpha \beta \gamma G^{\gamma-1}(x)\left(1-G^{\gamma}(x)\right)^{\alpha-1}}{1-\left(1-G^{\gamma}(x)\right)^{\alpha}} .
\end{gathered}
$$

Note that when $\gamma=1$ we obtain the exponentiated generalized family of distributions that was introduced by Cordeiro et al. (2013). The GIKw-G family is easily simulated by inverting (1.3) as follows: if $\mathrm{u}$ has a uniform $U(0,1)$ distribution, then the solution of the nonlinear equation is given by

$$
Q_{x}(u)=G^{-1}\left\{1-\left(1-u^{\frac{1}{\beta}}\right)^{\frac{1}{\alpha}}\right\}^{\frac{1}{\gamma}} .
$$

The rest of the paper is organized as follows: In Section 2, four special models of the new family are given with their plots of density and hazard rate functions. In Section 3 , Structural Properties are discussed while in Section 4, parameter estimation and a Monte Carlo simulation study are presented. In Section 5, the proposed GIKw-G is applied to some real-life data and also compared with other well-known distributions.

\section{Some Special Models of the GIKw-G Family}

In this section, we give some examples of the GIKw-G family of distributions, namely: the generalized inverted Kumaraswamy-uniform, generalized inverted Kumaraswamy-Weibull, generalized inverted Kumaraswamy-log logistic and generalized inverted Kumaraswamy-Lomax distributions.

\subsection{Generalized inverted Kumaraswamy-uniform (GIKw-U) distribution}

The pdf of the GIKw-U is derived from (1.4), by taking $g(x ; \theta)=\frac{1}{\theta} \quad, 0<x<\theta$, and $G(x ; \theta)=\frac{x}{\theta}$ as follows:

$$
f(x)=\frac{\alpha \beta \gamma}{\theta}\left(\frac{x}{\theta}\right)^{\gamma-1}\left[1-\left(\frac{x}{\theta}\right)^{\gamma}\right]^{\alpha-1}\left\{1-\left[1-\left(\frac{x}{\theta}\right)^{\gamma}\right]^{\alpha}\right\}^{\beta-1} .
$$


The corresponding cdf takes the following form

$$
F(x)=\left\{1-\left[1-\left(\frac{x}{\theta}\right)^{\gamma}\right]^{\alpha}\right\}^{\beta}
$$

The plots of density and hazard rate functions of the GIKw-U are presented in Figure 1. As seen in 1(a), the density function has reversed $\mathrm{J}$ and upside-down bathtub shapes. Figure 1(b) shows that the hazard rate function gives increasing and bathtub shapes.

(a)

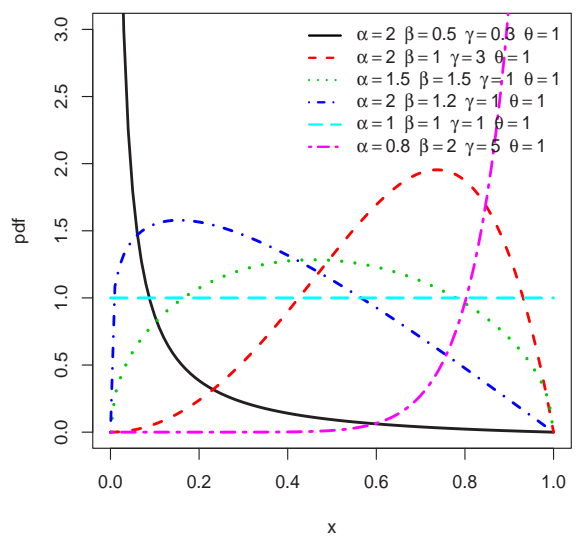

(b)

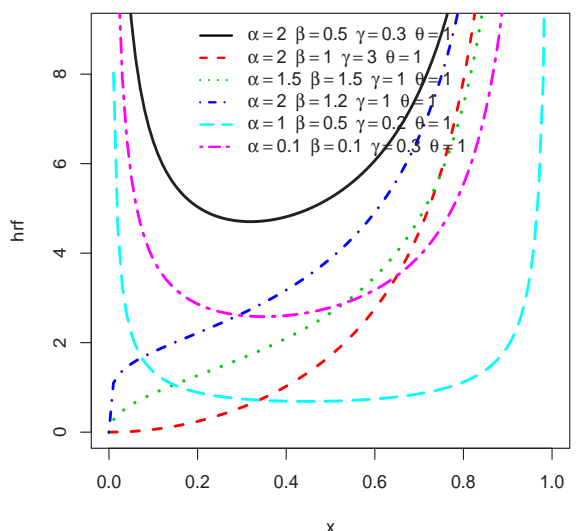

Figure 1: Plots of pdf and hrf for the GIKw-U distribution with several values of parameters

\subsection{Generalized inverted Kumaraswamy-Weibull (GIKw-W) distribution}

We consider the Weibull distribution with scale and shape parameters $a, b>0$. The pdf and cdf are $g(x)=a b x^{b-1} e^{-a x^{b}}$ and $G(x)=1-e^{-a x^{b}}$, respectively. Then, the pdf of the GIKw-W is given by

$f(x)=a b \alpha \beta \gamma x^{b-1} e^{-a x^{b}}\left(1-e^{-a x^{b}}\right)^{\gamma-1}\left[1+\left(1-e^{-a x^{b}}\right)^{\gamma}\right]^{\alpha-1}\left\{1-\left[1+\left(1-e^{-a x^{b}}\right)^{\gamma}\right]^{\alpha}\right\}^{\beta-1}$,

The corresponding cdf takes the following form

$$
F(x)=\left\{1-\left[1+\left(1-e^{-a x^{b}}\right)^{\gamma}\right]^{\alpha}\right\}^{\beta}
$$

Figure 2 shows the plots of density and hazard functions of GIKw-W distribution. The density function in Figure 2(a) has reversed J, left, right skewed and symmetrical shapes. The hrf in Figure 2(b) gives bathtub, upside-down bathtub and increasing shapes.

\subsection{Generalized inverted Kumaraswamy-Log logistic (GIKw-LL) distribution}

We consider the log-logistic distribution with scale parameter $\theta>0$. The pdf and cdf are given by $g(x)=\frac{\theta x^{\theta-1}}{\left(1+x^{\theta}\right)^{2}}$ and $G(x)=\frac{x^{\theta}}{1+x^{\theta}}$, respectively. Then, the cdf of the GIKw-LL distribution is given 
(a)

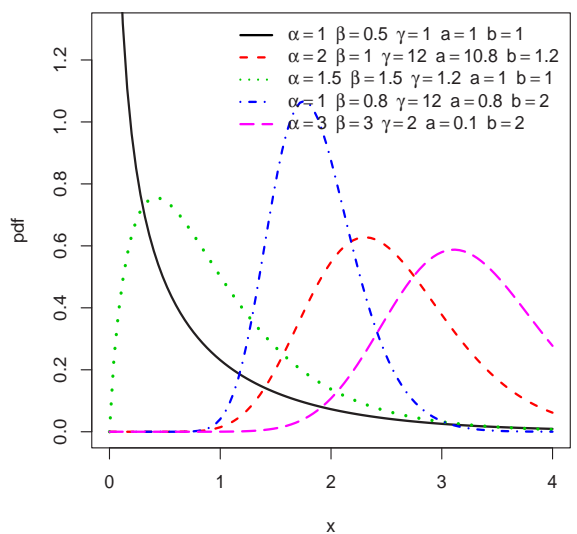

(b)

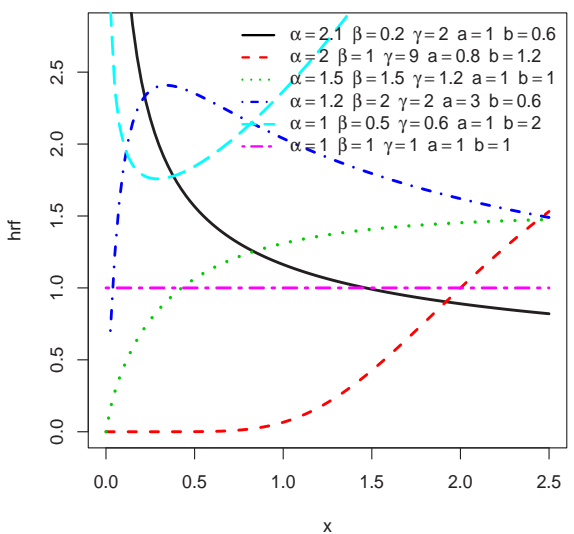

Figure 2: Plots of pdf and hrf for the GIKw-W distribution with several values of parameters

by

$$
f(x)=\alpha \beta \gamma \frac{\theta x^{\theta-1}}{\left(1+x^{\theta}\right)^{2}}\left(\frac{x^{\theta}}{1+x^{\theta}}\right)^{\gamma-1}\left[1-\left(\frac{x^{\theta}}{1+x^{\theta}}\right)^{\gamma}\right]^{\alpha-1}\left\{1-\left[1-\left(\frac{x^{\theta}}{1+x^{\theta}}\right)^{\gamma}\right]^{\alpha}\right\}^{\beta-1}
$$

The corresponding cdf takes the following form

$$
F(x)=\left\{1-\left[1-\left(\frac{x^{\theta}}{1+x^{\theta}}\right)^{\gamma}\right]^{\alpha}\right\}^{\beta}
$$

The plots of density and hazard rate functions of GIKw-LL are given in Figure 3. The density in Figure 3(a) gives reversed J, nearly symmetrical and positively skewed shapes. As seen in Figure $3(\mathrm{~b})$, the hazard rate function has the bathtub, upside-down bathtub, increasing and decreasing shapes.

\subsection{Generalized inverted Kumaraswamy-Lomax (GIKw-Lx) distribution}

We consider the Lomax distribution with scale parameter $a, b>0$. The pdf and cdf are $g(x)=$ $\frac{a}{b}\left(1+\frac{x}{b}\right)^{a-1}$ and $g(x)=1-\left(1+\frac{x}{b}\right)^{a}$, respectively. Then, the pdf of GIKw-L is given by

$$
\begin{aligned}
f(x)= & \alpha \beta \gamma \frac{a}{b}\left(1+\frac{x}{b}\right)^{a-1}\left[1-\left(1+\frac{x}{b}\right)^{a}\right]^{\gamma-1}\left\{1-\left[1-\left(1+\frac{x}{b}\right)^{a}\right]^{\gamma}\right\}^{\alpha-1} \\
& {\left[1-\left\{1-\left[1-\left(1+\frac{x}{b}\right)^{a}\right]^{\gamma}\right\}^{\alpha}\right]^{\beta-1} }
\end{aligned}
$$

The corresponding cdf takes the following form

$$
F(x)=\left[1-\left\{1-\left[1-\left(1+\frac{x}{b}\right)^{a}\right]^{\gamma}\right\}^{\alpha}\right]^{\beta}
$$


(a)

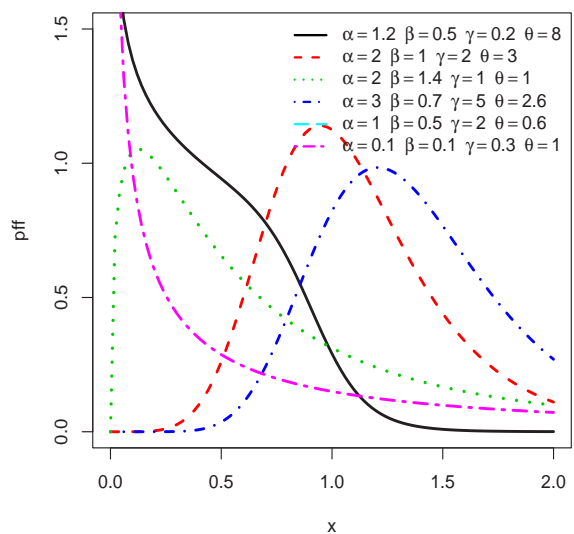

(b)

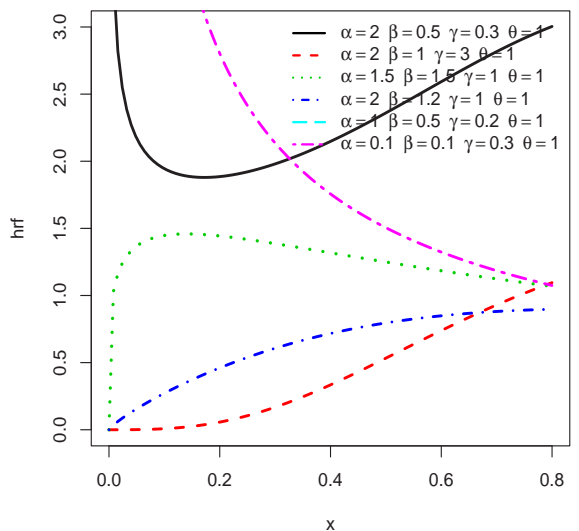

Figure 3: Plots of pdf and hrf for the GIKw-LL distribution with several values of parameters

The plots of density and hazard rate functions of the GIKw-L are given in Figure 4. Figure 4(a) shows that reversed $\mathrm{J}$ and positively skewed shapes are possible for the density function. Figure 4(b) also shows that the hazard rate function has upside-down bathtub, increasing and decreasing shapes.

(a)

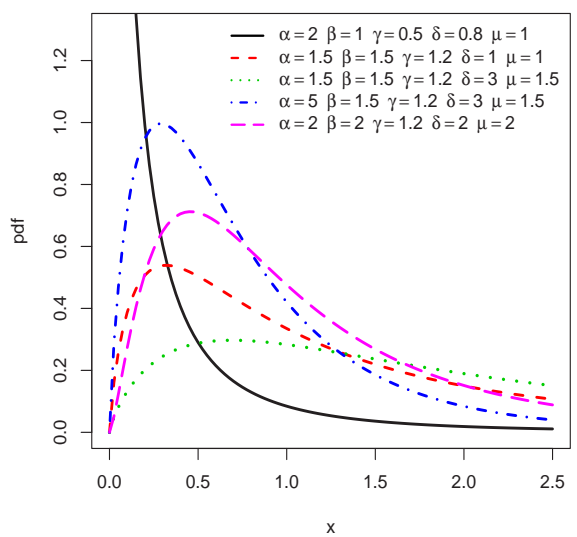

(b)

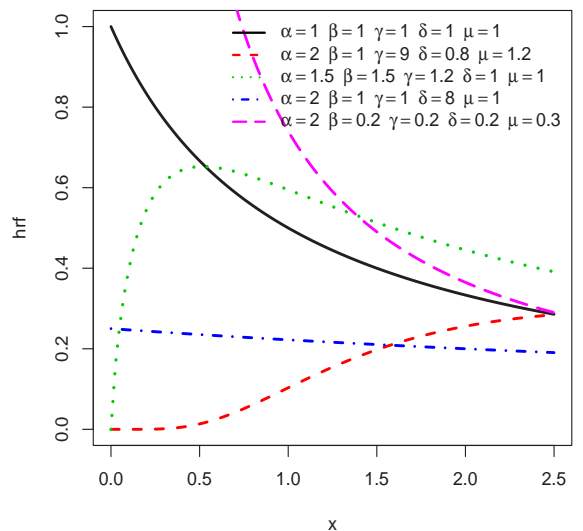

Figure 4: Plots of pdf and hrf for the GIKw-L distribution with several values of parameters

\section{Structural Properties}

The several types of moments of a random variable are important especially in applied work. Some of the most important features and characteristics of a distribution can be studied through moments, e.g., tendency, dispersion, skewness and kurtosis, mean deviations, Bonferroni and Lorenz curves, etc. In this section, we will provide the expansions of GIKw-G densities, $r$ th moment, sth 
incomplete moment, moment generating function and mean deviations.

\subsection{Useful expansions}

Here, the infinite mixture representations for the cdf and pdf of the GIKw-G family are given in terms of baseline densities. Consider following series expansion

$$
(1-z)^{b}=\sum_{j=0}^{\infty}\left(\begin{array}{l}
b \\
j
\end{array}\right)(-1)^{j} z^{j}
$$

Using the series expansion in (3.1), the equation (1.3) becomes

$$
F(x)=\sum_{j=0}^{\infty}\left(\begin{array}{c}
\beta \\
j
\end{array}\right)(-1)^{j} \sum_{i=0}^{\infty}\left(\begin{array}{c}
\alpha j \\
i
\end{array}\right)(-1)^{i} G^{\gamma i}
$$

where $G^{\gamma i}=\sum_{q=0}^{\infty} \sum_{q=k}^{\infty}\left(\begin{array}{c}\gamma i \\ k\end{array}\right)\left(\begin{array}{c}k \\ q\end{array}\right)(-1)^{q+k} G^{q}(x)$. Now the above expression can be written as

$$
F(x)=\sum_{j=0}^{\infty} \sum_{i=0}^{\infty}\left(\begin{array}{c}
\beta \\
j
\end{array}\right)\left(\begin{array}{c}
\alpha j \\
i
\end{array}\right)(-1)^{i+j} \sum_{q=0}^{\infty} \sum_{q=k}^{\infty}\left(\begin{array}{c}
\gamma i \\
k
\end{array}\right)\left(\begin{array}{c}
k \\
q
\end{array}\right)(-1)^{q+k} G^{q}(x)
$$

. Rewriting the above expression, we obtain an expansion for the cdf of the GIKw-G family

$$
F(x)=\sum_{q=0}^{\infty} a_{q} H_{q}(x),
$$

where $a_{q}=\sum_{j=0}^{\infty} \sum_{i=0}^{\infty}\left(\begin{array}{c}\beta \\ j\end{array}\right)\left(\begin{array}{c}\alpha j \\ i\end{array}\right)(-1)^{i+j} \sum_{q=k}^{\infty}\left(\begin{array}{c}\gamma i \\ k\end{array}\right)\left(\begin{array}{c}k \\ q\end{array}\right)(-1)^{q+k}$ and $H_{q}(x)=G^{q}(x)$.

Similarly, we have an expansion for the pdf of the GIKw-G family as

$$
f(x)=\sum_{q=0}^{\infty} a_{q} h_{q-1}(x)
$$

where $h_{q-1}(x)=q \times g(x) G^{q-1}(x)$.

\subsection{Moments and moment generating function}

Moments are necessary and important in any statistical analysis, especially in applications. It can be used to study the most important features and characteristics of a distribution (e.g., tendency, dispersion, skewness and kurtosis). The $r$ th moment of GIKw-G family is given by

$$
\mu_{r}^{\prime}=\int_{0}^{\infty} x^{r} f(x) d x
$$


. Using the infinite mixture representation of the pdf in equation (3.3), we have

$$
\mu_{r}^{\prime}=\sum_{q=0}^{\infty} a_{q} \Delta_{r}^{\prime}
$$

where $\Delta_{r}^{\prime}=\int_{0}^{\infty} x^{r} h_{q-1}(x) d x$

Similarly, the sth incomplete moment of GIKw-G family is defined as

$$
\mu_{s}^{\prime}=\int_{0}^{x} x^{s} f(x) d x
$$

From the infinite mixture representation of pdf in equation (3.3), we get

$$
\mu_{s}^{\prime}=\sum_{q=0}^{\infty} a_{q} T_{s}^{\prime}(x)
$$

where $T_{s}^{\prime}(x)=\int_{0}^{x} x^{s} h_{q-1}(x) d x$.

Now, we define the moment generating function of GIKw-G family as

$$
M_{0}(t)=\int_{0}^{\infty} e^{t x} f(x) d x
$$

From the infinite mixture representation of pdf in equation (3.3), we obtain

$$
M_{0}(t)=\sum_{q=0}^{\infty} a_{q} M_{q-1}(t)
$$

where $M_{q-1}(t)=\int_{0}^{x} e^{t x} h_{q-1}(x) d x$.

Note that the integrals $\Delta_{r}^{\prime}, T_{s}^{\prime}(x)$ and $M_{q-1}(t)$ depend only for any choice of baseline distribution. The mean deviations of GIKw-G family is obtained by

$$
\delta_{1}=2 \mu F(\mu)-2 J(\mu), \quad \delta_{2}=\mu-2 J(\widetilde{x})
$$

where $\mu=$ mean $=E(X), \widetilde{x}=$ median $=Q_{x}(0.5)$ and $j(c)=\int_{0}^{x} x f(x) d x$. Using the infinite mixture representation of pdf in equation (3.3), we get

$$
j(c)=\sum_{q=0}^{\infty} a_{q} S_{q-1}^{\prime}(c)
$$

where $S_{q-1}^{\prime}(c)=\int_{0}^{c} x h_{q-1}(x) d x$ the integral depends on $G(x)$ and $g(x)$. 


\subsection{Entropies}

The entropy of a random variable $\mathrm{X}$ is a measure of variation of uncertainty and has been used in many fields such as physics, engineering, and economics. In this section, we will consider two well known entropy measures, Reǹyi and Shannon entropies. According to Reǹyi (1961), the Reǹyi entropy is defined by

$$
I_{R}=\frac{1}{1-R} \log \int_{0}^{\infty} f^{R}(x) d x
$$

where $f^{R}(x)=\left[\alpha \beta \gamma g(x) G^{\gamma-1}(x)\left(1-G^{\gamma}(x)\right)^{\alpha-1}\left[1-\left(1-G^{\gamma}(x)\right)^{\alpha}\right]^{\beta-1}\right]^{R}$.

Using series expansion in equation (3.1), we have

$$
f^{R}(x)=\sum_{i=0}^{\infty} w_{i}(j) g_{\gamma(i+R)-R}(x)
$$

Here, we define $w_{i}(j)=(\alpha \beta \gamma)^{R} \sum_{j=0}^{\infty}\left(\begin{array}{c}R(\beta-1) \\ j\end{array}\right)\left(\begin{array}{c}\alpha(j+R)-R \\ i\end{array}\right) \frac{(-1)^{i+j}}{\gamma(i+R)-R+1}$

and $g_{\gamma(i+R)-R}(x)=[\gamma(i+R)-R+1] g^{R}(x) G^{\gamma(i+R)-R}(x)$.

Now equation (3.5) becomes

$$
I_{R}=\frac{1}{1-R} \log \left[\sum_{i=0}^{\infty} w_{i}(j) \int_{0}^{\infty} g_{\gamma(i+R)-R}(x) d x\right]
$$

The above integral depends on $G(x)$ and $g(x)$.

The Shannon entropy of a random variable X is defined by Shannon (1948) as

$$
\eta_{x}=-E[\log f(x)]
$$

From equation (1.4), we have

$$
\eta_{x}=-E\left[\log \gamma+\log g(x)+(\gamma-1) \log \left(1-G^{\gamma}(x)\right)+\log r\left\{\frac{G^{\gamma}(x)}{1-G^{\gamma}(x)}\right\}\right]
$$

Let $T=\frac{G^{\gamma}(x)}{1-G^{\gamma}(x)}$ then $G(x)=\left[\frac{T}{1+T}\right]^{\frac{1}{\gamma}}$ and $1-G^{\gamma}(x)=\left[\frac{1}{1+T}\right]$.

Now equation (3.6) becomes

$$
\eta_{x}=-E\left[\log \gamma+\log g(x)+\left(1-\frac{1}{\gamma}\right) \log T+\left(1+\frac{1}{\gamma}\right) \log (1+T)+\log r(T)\right]
$$

After some algebra we have

$$
\begin{aligned}
\eta_{x} & =\eta_{g}+\eta_{T}-\log \gamma-\left(1-\frac{1}{\gamma}\right) \sum_{j=0}^{\infty} \frac{(-1)^{j}}{j !} \frac{\mathbf{B}\left(1-\frac{j}{\alpha}, \beta\right)}{\Gamma(1-j)}\left[\Gamma^{\prime}(1)-\psi(1-j)\right] \\
& +\frac{1}{\alpha}\left(1+\frac{1}{\gamma}\right)\left[\Gamma^{\prime}(1)-\psi(\beta+1)\right]
\end{aligned}
$$

where $\eta_{T}$ is the Shannon entropy of IKw distribution and $\eta_{g}$ is the Shannon entropy of baseline distribution. where $\psi(a)=\frac{\Gamma^{\prime}(a)}{\Gamma(a)}$ and $\Gamma^{\prime}(1)$ is Euler's constant. 


\subsection{Order Statistics}

Order statistics have been extensively applied in many fields of statistics, such as reliability and life testing. They enter in the problems of estimation and hypothesis tests in a variety of ways. We now discuss some properties of the order statistics for the GIKw-G family. Let $X_{i: n}$ denote the $i$ th order statistic from a random sample $X_{1}, \ldots, X_{n}$ from the GIKw-G family. Then, the density function of $i$ th order statistic is given by

$$
f_{i: n}(x)=\frac{n !}{(i-1) !(n-i) !} f(x) F^{i-1}(x)[1-F(x)]^{n-i}
$$

Using series expansion in equation (3.1), we have

$$
f_{i: n}(x)=\frac{n !}{(i-1) !(n-i) !} \sum_{j=0}^{n-i}\left(\begin{array}{c}
n-i \\
j
\end{array}\right)(-1)^{j} f(x) F^{j+i-1}(x)
$$

Consider $f(x) F^{j+i-1}(x)$, using infinite mixture representation in equations (3.2) and (3.3), we obtain

$$
\left[\sum_{q=0}^{\infty} a_{q} h_{q-1}(x)\right] \times\left[\sum_{m=0}^{\infty} a_{m} H_{m}(x)\right]^{j+i-1}
$$

From power series raised to a positive power expansion $\left[\sum_{k=0}^{\infty} a_{k} G^{k}(x)\right]^{r}=\sum_{k=0}^{\infty} d_{k: r} G^{k}(x)$ we have

$$
\left[\sum_{q=0}^{\infty} a_{q} q g(x) G^{q}(x)\right] \times \sum_{m=0}^{\infty} d_{m: j+i-1} G^{m}(x)
$$

After simplification, we have

$$
\left[\sum_{q=0}^{\infty} \sum_{m=0}^{\infty} a_{q} d_{m: j+i-1} q g(x) G^{q+r}(x)\right]
$$

Now equation (3.8) becomes

$$
f_{i: n}(x)=\frac{n !}{(i-1) !(n-i) !} \sum_{j=0}^{n-i}\left(\begin{array}{c}
n-i \\
j
\end{array}\right)(-1)^{j} \sum_{q=0}^{\infty} \sum_{m=0}^{\infty} a_{q} d_{m: j+i-1} q g(x) G^{q+r}(x)
$$

Rewriting the equation, the density function of $i$ th order statistic is given by

$$
f_{i: n}(x)=\sum_{j=0}^{n-i} \sum_{q=0}^{\infty} \sum_{m=0}^{\infty} V_{j}(q, m) h_{q+m-1}(x)
$$

, where $V_{j}(q, m)=\frac{n !}{(i-1) !(n-i) !}\left(\begin{array}{c}n-i \\ j\end{array}\right) \frac{(-1)^{j} a_{q} d_{m: j+i-1} q}{q+m}$

and $h_{q+m-1}(x)=(q+m) g(x) G^{q+m-1}$. 


\section{Estimation and Simulation}

\subsection{Estimation}

Here, we consider the estimation of the unknown parameters of GIKw-G family by the maximum likelihood method for the complete samples. The maximum likelihood estimates (MLEs) enjoy desirable properties that can be used when constructing confidence intervals and deliver simple approximations that work well in finite samples. The resulting approximation for the MLEs in distribution theory is easily handled either analytically or numerically. Let $x_{1}, \ldots, x_{n}$ be a sample of size $\mathrm{n}$ from GIKw-G family given in (1.3). The log-likelihood function of GIKw-G family for the vector of parameter $\Theta=(\alpha, \beta, \gamma, \xi)^{T}$ is given by

$$
\begin{aligned}
\ell(\Theta) \quad & =n \log (\alpha \beta \gamma)+\sum_{i=1}^{n} \log g\left(x_{i}, \xi\right)+(\gamma-1) \sum_{i=1}^{n} \log G\left(x_{i}, \xi\right)+(\alpha-1) \sum_{i=1}^{n} \log \left[1-G^{\gamma}\left(x_{i}, \xi\right)\right] \\
& +(\beta-1) \sum_{i=1}^{n} \log \left[1-\left\{1-G^{\gamma}\left(x_{i}, \xi\right)\right\}^{\alpha}\right]
\end{aligned}
$$

The components of score vector $U=\left(U_{\beta}, U_{\alpha}, U_{\gamma}, U_{\xi}\right)^{T}$ are given by

$$
\begin{aligned}
U_{\beta}= & \frac{n}{\beta}+\sum_{i=1}^{n} \log \left[1-\left\{1-G^{\gamma}\left(x_{i}, \xi\right)\right\}^{\alpha}\right] \\
U_{\alpha}= & \frac{n}{\alpha}+\sum_{i=1}^{n} \log \left[1-G^{\gamma}\left(x_{i}, \xi\right)\right]+(\beta-1) \sum_{i=1}^{n}\left[\frac{\left\{1-G^{\gamma}\left(x_{i}, \xi\right)\right\}^{\alpha} \log \left\{1-G^{\gamma}\left(x_{i}, \xi\right)\right\}}{1-\left\{1-G^{\gamma}\left(x_{i}, \xi\right)\right\}^{\alpha}}\right] \\
U_{\gamma}= & \frac{n}{\gamma}+\sum_{i=1}^{n} \log G\left(x_{i}, \xi\right)+(\alpha-1) \sum_{i=1}^{n}\left[\frac{G^{\gamma}\left(x_{i}, \xi\right) \log G\left(x_{i}, \xi\right)}{1-G^{\gamma}\left(x_{i}, \xi\right)}\right] \\
& +(\beta-1) \alpha \sum_{i=1}^{n}\left[\frac{\left\{1-G^{\gamma}\left(x_{i}, \xi\right)\right\}^{\alpha-1} G^{\gamma}\left(x_{i}, \xi\right) \log G\left(x_{i}, \xi\right)}{1-\left\{1-G^{\gamma}\left(x_{i}, \xi\right)\right\}^{\alpha}}\right] \\
U_{\xi}= & \sum_{i=1}^{n}\left[\frac{g^{\xi}\left(x_{i}, \xi\right)}{g\left(x_{i}, \xi\right)}\right]+(\gamma-1) \sum_{i=1}^{n}\left[\frac{G^{\xi}\left(x_{i}, \xi\right)}{G\left(x_{i}, \xi\right)}\right]-\gamma(\alpha-1) \sum_{i=1}^{n}\left[\frac{G^{\gamma-1}\left(x_{i}, \xi\right) G^{\xi}\left(x_{i}, \xi\right)}{1-G^{\gamma}\left(x_{i}, \xi\right)}\right] \\
& -\alpha \gamma(\beta-1) \sum_{i=1}^{n}\left[\frac{\left\{1-G^{\gamma}\left(x_{i}, \xi\right)\right\}^{\alpha-1} G^{\gamma-1}\left(x_{i}, \xi\right) G^{\xi}\left(x_{i}, \xi\right)}{1-\left\{1-G^{\gamma}\left(x_{i}, \xi\right)\right\}^{\alpha}}\right]
\end{aligned}
$$

Setting $U_{\beta}, U_{\alpha}, U_{\gamma}$ and $U_{\xi}$ equal to zero and solving these equations simultaneously yields the the MLEs. These equations cannot be solved analytically, and analytical softwares are required to solve them numerically.

\subsection{Simulation study}

In this section, an extensive numerical investigation will be carried out to evaluate the performance of the MLEs for the GIKw-W model. Performance of estimators is evaluated through their biases, and mean square errors (MSEs) for different sample sizes. A numerical study is performed using 
Mathematica (9) software. Different sample sizes ( $\mathrm{n}=30,50,75,100,200$ and 300) and different values of parameters are considered. The experiment is repeated 3000 times. In each experiment, the estimates of the parameters are obtained by maximum likelihood method. The means, MSEs and biases for the different estimators are reported from these experiments.

The values in Table 1 indicate that the estimates are quite stable and, more importantly, are close to the true values for the these sample sizes. From Table 1, it is observed that in general the standard deviation decreases as $\mathrm{n}$ increases. The simulation study shows that the maximum likelihood method is appropriate for estimating the GIKw-W parameters. In fact, the MSEs of the parameters tend to be closer to the true parameter values when $\mathrm{n}$ increases. This fact supports that the asymptotic normal distribution provides an adequate approximation to the finite sample distribution of the MLEs. The normal approximation can be often times improved by using bias adjustments to these estimators.

Table 1: The parameter estimations from the GIKw-W distribution using the maximum likelihood method

\begin{tabular}{cccccccccc}
\hline $\mathrm{n}$ & Par & Init & MLE & Bias & MSE & Init & MLE & Bias & MSE \\
\hline \multirow{6}{*}{50} & $\gamma$ & 2 & 2.0915 & 0.0915 & 0.2296 & 2 & 2.0905 & 0.0905 & 0.2085 \\
& $\alpha$ & 2 & 2.0580 & 0.0580 & 0.1427 & 2 & 2.0488 & 0.0488 & 0.1308 \\
& $\beta$ & 1.5 & 1.5316 & 0.0316 & 0.0522 & 1.5 & 1.5330 & 0.0330 & 0.0485 \\
& $\mathrm{a}$ & 2 & 2.0752 & 0.0752 & 11.7134 & 2 & 2.1234 & 0.1234 & 0.4287 \\
& $\mathrm{~b}$ & 0.5 & 0.5293 & 0.0293 & 0.0200 & 2 & 2.1119 & 0.1119 & 0.2870 \\
\hline \multirow{6}{*}{75} & $\gamma$ & 2 & 2.0679 & 0.0679 & 0.1361 & 2 & 2.0559 & 0.0559 & 0.1356 \\
& $\alpha$ & 2 & 2.0311 & 0.0311 & 0.0882 & 2 & 2.0430 & 0.0430 & 0.0897 \\
& $\beta$ & 1.5 & 1.5255 & 0.0255 & 0.0335 & 1.5 & 1.5196 & 0.0196 & 0.0332 \\
& $\mathrm{a}$ & 2 & 2.0775 & 0.0775 & 0.2373 & 2 & 2.0952 & 0.0952 & 0.2366 \\
& $\mathrm{~b}$ & 0.5 & 0.5204 & 0.0204 & 0.0114 & 2 & 2.0679 & 0.0679 & 0.1647 \\
\hline \multirow{4}{*}{100} & $\gamma$ & 2 & 2.0434 & 0.0434 & 0.0940 & 2 & 2.0433 & 0.0433 & 0.0945 \\
& $\alpha$ & 2 & 2.0290 & 0.0290 & 0.0625 & 2 & 2.0259 & 0.0259 & 0.0610 \\
& $\beta$ & 1.5 & 1.5161 & 0.0161 & 0.0239 & 1.5 & 1.5155 & 0.0155 & 0.0241 \\
& $\mathrm{a}$ & 2 & 2.0630 & 0.0630 & 0.1512 & 2 & 2.0598 & 0.0598 & 0.1523 \\
& $\mathrm{~b}$ & 0.5 & 0.5136 & 0.0136 & 0.0074 & 2 & 2.0526 & 0.0526 & 0.1197 \\
\hline \multirow{3}{*}{200} & $\gamma$ & 2 & 2.0190 & 0.0190 & 0.0425 & 2 & 2.0227 & 0.0227 & 0.0426 \\
& $\alpha$ & 2 & 2.0164 & 0.0164 & 0.0302 & 2 & 2.0122 & 0.0122 & 0.0293 \\
& $\beta$ & 1.5 & 1.5071 & 0.0071 & 0.0115 & 1.5 & 1.5089 & 0.0089 & 0.0115 \\
& $\mathrm{a}$ & 2 & 2.0335 & 0.0335 & 0.0679 & 2 & 2.0274 & 0.0274 & 0.0663 \\
& $\mathrm{~b}$ & 0.5 & 0.5063 & 0.0063 & 0.0034 & 2 & 2.0273 & 0.0273 & 0.0547 \\
\hline \multirow{6}{*}{300} & $\gamma$ & 2 & 2.0128 & 0.0128 & 0.0272 & 2 & 2.0155 & 0.0155 & 0.0282 \\
& $\alpha$ & 2 & 2.0070 & 0.0070 & 0.0194 & 2 & 2.0104 & 0.0104 & 0.0200 \\
& $\beta$ & 1.5 & 1.5045 & 0.0045 & 0.0074 & 1.5 & 1.5066 & 0.0066 & 0.0077 \\
& $\mathrm{a}$ & 2 & 2.0173 & 0.0173 & 0.0426 & 2 & 2.0211 & 0.0211 & 0.0433 \\
& $\mathrm{~b}$ & 0.5 & 0.5031 & 0.0031 & 0.0021 & 2 & 2.0199 & 0.0199 & 0.0351 \\
\hline & & & & & & & & &
\end{tabular}


Continued of Table 1

\begin{tabular}{|c|c|c|c|c|c|c|c|c|c|}
\hline $\mathrm{n}$ & Par & Init & MLE & Bias & MSE & Init & MLE & Bias & MSE \\
\hline \multirow{5}{*}{50} & $\gamma$ & 0.5 & 0.5216 & 0.0216 & 0.0133 & 1.5 & 1.5602 & 0.0602 & 0.1239 \\
\hline & $\alpha$ & 2 & 2.0570 & 0.0570 & 0.1301 & 2 & 2.0615 & 0.0615 & 0.1354 \\
\hline & $\beta$ & 1.5 & 1.5308 & 0.0308 & 0.0493 & 1.5 & 1.5265 & 0.0265 & 0.0496 \\
\hline & $\mathrm{a}$ & 2 & 2.0233 & 0.0233 & 0.0488 & 2 & 2.1006 & 0.1006 & 0.2491 \\
\hline & b & 2 & 2.1281 & 0.1281 & 0.3687 & 2 & 2.1099 & 0.1099 & 0.3171 \\
\hline \multirow{5}{*}{75} & $\gamma$ & 0.5 & 0.5135 & 0.0135 & 0.0078 & 1.5 & 1.5482 & 0.0482 & 0.0745 \\
\hline & $\alpha$ & 2 & 2.0339 & 0.0339 & 0.0884 & 2 & 2.0331 & 0.0331 & 0.0833 \\
\hline & $\beta$ & 1.5 & 1.5188 & 0.0188 & 0.0310 & 1.5 & 1.5241 & 0.0241 & 0.0325 \\
\hline & $\mathrm{a}$ & 2 & 2.0115 & 0.0115 & 0.0347 & 2 & 2.0543 & 0.0543 & 0.1424 \\
\hline & $\mathrm{b}$ & 2 & 2.0753 & 0.0753 & 0.1946 & 2 & 2.0867 & 0.0866 & 0.1949 \\
\hline \multirow{5}{*}{100} & $\gamma$ & 0.5 & 0.5121 & 0.0121 & 0.0058 & 1.5 & 1.5321 & 0.0321 & 0.0509 \\
\hline & $\alpha$ & 2 & 2.0300 & 0.0299 & 0.0615 & 2 & 2.0255 & 0.0255 & 0.0606 \\
\hline & $\beta$ & 1.5 & 1.5192 & 0.0192 & 0.0234 & 1.5 & 1.5157 & 0.0157 & 0.0234 \\
\hline & $\mathrm{a}$ & 2 & 2.0141 & 0.0141 & 0.0248 & 2 & 2.0421 & 0.0421 & 0.1029 \\
\hline & $\mathrm{b}$ & 2 & 2.0704 & 0.0704 & 0.1417 & 2 & 2.0565 & 0.0565 & 0.1276 \\
\hline \multirow{5}{*}{200} & $\gamma$ & 0.5 & 0.5048 & 0.0048 & 0.0027 & 1.5 & 1.5162 & 0.0162 & 0.0253 \\
\hline & $\alpha$ & 2 & 2.0173 & 0.0173 & 0.0322 & 2 & 2.0176 & 0.0176 & 0.0306 \\
\hline & $\beta$ & 1.5 & 1.5073 & 0.0073 & 0.0114 & 1.5 & 1.5084 & 0.0084 & 0.0119 \\
\hline & $\alpha$ & 2 & 2.0077 & 0.0077 & 0.0134 & 2 & 2.0265 & 0.0265 & 0.0497 \\
\hline & $\mathrm{b}$ & 2 & 2.0264 & 0.0264 & 0.0609 & 2 & 2.0313 & 0.0313 & 0.0611 \\
\hline \multirow{5}{*}{300} & $\gamma$ & 0.5 & 0.5019 & 0.0019 & 0.0018 & 1.5 & 1.5152 & 0.0152 & 0.0154 \\
\hline & $\alpha$ & 2 & 2.0107 & 0.0107 & 0.0198 & 2 & 2.0063 & 0.0063 & 0.0185 \\
\hline & $\beta$ & 1.5 & 1.5017 & 0.0017 & 0.0077 & 1.5 & 1.5089 & 0.0089 & 0.0075 \\
\hline & $\mathrm{a}$ & 2 & 2.0042 & 0.0042 & 0.0083 & 2 & 2.0101 & 0.0101 & 0.0290 \\
\hline & $\mathrm{b}$ & 2 & 2.0116 & 0.0116 & 0.0404 & 2 & 2.0273 & 0.0273 & 0.0381 \\
\hline
\end{tabular}


Continued of Table 1

\begin{tabular}{|c|c|c|c|c|c|c|c|c|c|}
\hline $\mathrm{n}$ & Par & Init & MLE & Bias & MSE & Init & MLE & Bias & MSE \\
\hline \multirow{5}{*}{50} & $\gamma$ & 2 & 2.0216 & 0.0216 & 0.0377 & 2 & 2.0702 & 0.0702 & 0.1469 \\
\hline & $\alpha$ & 0.5 & 0.5148 & 0.0148 & 0.0085 & 1.5 & 1.5481 & 0.0481 & 0.0817 \\
\hline & $\beta$ & 1.5 & 1.5336 & 0.0336 & 0.0508 & 1.5 & 1.5350 & 0.0350 & 0.0495 \\
\hline & $\mathrm{a}$ & 2 & 2.0929 & 0.0929 & 0.2416 & 2 & 2.1382 & 0.1382 & 0.4421 \\
\hline & $\mathrm{b}$ & 2 & 2.1107 & 0.1107 & 0.2649 & 2 & 2.1004 & 0.1003 & 0.2074 \\
\hline \multirow{5}{*}{75} & $\gamma$ & 2 & 2.0142 & 0.0142 & 0.0241 & 2 & 2.0422 & 0.0422 & 0.0921 \\
\hline & $\alpha$ & 0.5 & 0.5090 & 0.0090 & 0.0053 & 1.5 & 1.5306 & 0.0306 & 0.0503 \\
\hline & $\beta$ & 1.5 & 1.5221 & 0.0221 & 0.0313 & 1.5 & 1.5205 & 0.0205 & 0.0318 \\
\hline & $\mathrm{a}$ & 2 & 2.0553 & 0.0552 & 0.1382 & 2 & 2.0844 & 0.0843 & 0.2127 \\
\hline & $\mathrm{b}$ & 2 & 2.0708 & 0.0708 & 0.1574 & 2 & 2.0567 & 0.0567 & 0.1275 \\
\hline \multirow{5}{*}{100} & $\gamma$ & 2 & 2.0105 & 0.0105 & 0.0191 & 2 & 2.0358 & 0.0358 & 0.0700 \\
\hline & $\alpha$ & 0.5 & 0.5071 & 0.0071 & 0.0040 & 1.5 & 1.5206 & 0.0206 & 0.0355 \\
\hline & $\beta$ & 1.5 & 1.5168 & 0.0168 & 0.0245 & 1.5 & 1.5180 & 0.0180 & 0.0246 \\
\hline & $\mathrm{a}$ & 2 & 2.0435 & 0.0435 & 0.1038 & 2 & 2.0565 & 0.0565 & 0.1402 \\
\hline & $\mathrm{b}$ & 2 & 2.0526 & 0.0526 & 0.1184 & 2 & 2.0468 & 0.04687 & 0.0962 \\
\hline \multirow{5}{*}{200} & $\gamma$ & 2 & 2.0049 & 0.0049 & 0.0093 & 2 & 2.0185 & 0.0185 & 0.0314 \\
\hline & $\alpha$ & 0.5 & 0.5036 & 0.0036 & 0.0018 & 1.5 & 1.5074 & 0.0074 & 0.0167 \\
\hline & $\beta$ & 1.5 & 1.5079 & 0.0079 & 0.0115 & 1.5 & 1.5094 & 0.0094 & 0.0114 \\
\hline & $\mathrm{a}$ & 2 & 2.0215 & 0.0215 & 0.0453 & 2 & 2.0220 & 0.0220 & 0.0608 \\
\hline & $\mathrm{b}$ & 2 & 2.0271 & 0.0271 & 0.0535 & 2 & 2.0220 & 0.0220 & 0.0446 \\
\hline \multirow{5}{*}{300} & $\gamma$ & 2 & 2.0035 & 0.0035 & 0.0063 & 2 & 2.0072 & 0.0072 & 0.0206 \\
\hline & $\alpha$ & 0.5 & 0.5020 & 0.0020 & 0.0012 & 1.5 & 1.5090 & 0.0090 & 0.0105 \\
\hline & $\beta$ & 1.5 & 1.5054 & 0.0054 & 0.0077 & 1.5 & 1.5033 & 0.0033 & 0.0076 \\
\hline & $\beta$ & 2 & 2.0127 & 0.0127 & 0.0294 & 2 & 2.0221 & 0.0220 & 0.0381 \\
\hline & $\mathrm{b}$ & 2 & 2.0153 & 0.0153 & 0.0361 & 2 & 2.0127 & 0.0126 & 0.0289 \\
\hline
\end{tabular}


Continued of Table 1

\begin{tabular}{|c|c|c|c|c|c|c|c|c|c|}
\hline $\mathrm{n}$ & Par & Init & MLE & Bias & MSE & Init & MLE & Bias & MSE \\
\hline \multirow{5}{*}{50} & $\gamma$ & 2 & 2.0677 & 0.0676 & 0.1638 & 2 & 2.1000 & 0.1000 & 0.2439 \\
\hline & $\alpha$ & 2 & 2.0258 & 0.0258 & 0.0428 & 2 & 2.0811 & 0.0811 & 0.2101 \\
\hline & $\beta$ & 0.5 & 0.5108 & 0.0108 & 0.0059 & 2 & 2.0446 & 0.0446 & 0.0909 \\
\hline & $\mathrm{a}$ & 2 & 2.0540 & 0.0540 & 0.1050 & 2 & 2.2140 & 0.2140 & 1.1668 \\
\hline & $\mathrm{b}$ & 2 & 2.1049 & 0.1049 & 0.2696 & 2 & 2.1151 & 0.1151 & 0.2856 \\
\hline \multirow{5}{*}{75} & $\gamma$ & 2 & 2.0479 & 0.0478 & 0.1001 & 2 & 2.0570 & 0.0570 & 0.1322 \\
\hline & $\alpha$ & 2 & 2.0100 & 0.0100 & 0.0279 & 2 & 2.0519 & 0.0519 & 0.1092 \\
\hline & $\beta$ & 0.5 & 0.5076 & 0.0076 & 0.0038 & 2 & 2.0266 & 0.0266 & 0.0541 \\
\hline & $\mathrm{a}$ & 2 & 2.0257 & 0.0257 & 0.0652 & 2 & 2.1124 & 0.1124 & 0.2881 \\
\hline & $\mathrm{b}$ & 2 & 2.0662 & 0.0661 & 0.1534 & 2 & 2.0685 & 0.0685 & 0.1559 \\
\hline \multirow{5}{*}{100} & $\gamma$ & 2 & 2.0308 & 0.0308 & 0.0680 & 2 & 2.0479 & 0.0479 & 0.1035 \\
\hline & $\alpha$ & 2 & 2.0112 & 0.0112 & 0.0213 & 2 & 2.0379 & 0.0379 & 0.0830 \\
\hline & $\beta$ & 0.5 & 0.5049 & 0.0049 & 0.0027 & 2 & 2.0223 & 0.0223 & 0.0430 \\
\hline & $\mathrm{a}$ & 2 & 2.0241 & 0.0241 & 0.0493 & 2 & 2.0822 & 0.0822 & 0.2088 \\
\hline & $\mathrm{b}$ & 2 & 2.0461 & 0.0461 & 0.1043 & 2 & 2.0563 & 0.0563 & 0.1194 \\
\hline \multirow{5}{*}{200} & $\gamma$ & 2 & 2.0258 & 0.0258 & 0.0346 & 2 & 2.0265 & 0.0265 & 0.0445 \\
\hline & $\alpha$ & 2 & 2.0045 & 0.0045 & 0.0108 & 2 & 2.0153 & 0.0153 & 0.0382 \\
\hline & $\beta$ & 0.5 & 0.5047 & 0.0047 & 0.0014 & 2 & 2.0135 & 0.0134 & 0.0198 \\
\hline & $\mathrm{a}$ & 2 & 2.0096 & 0.0096 & 0.0246 & 2 & 2.0330 & 0.0330 & 0.0849 \\
\hline & $\mathrm{b}$ & 2 & 2.0360 & 0.0360 & 0.0505 & 2 & 2.0316 & 0.0316 & 0.0522 \\
\hline \multirow{5}{*}{300} & $\gamma$ & 2 & 2.0056 & 0.0056 & 0.0217 & 2 & 2.0181 & 0.0181 & 0.0302 \\
\hline & $\alpha$ & 2 & 2.0042 & 0.0042 & 0.0072 & 2 & 2.0105 & 0.0105 & 0.0253 \\
\hline & $\beta$ & 0.5 & 0.5007 & 0.0007 & 0.0009 & 2 & 2.0095 & 0.0095 & 0.0136 \\
\hline & $\mathrm{a}$ & 2 & 2.0093 & 0.0093 & 0.0159 & 2 & 2.0225 & 0.0225 & 0.0542 \\
\hline & $\mathrm{b}$ & 2 & 2.0083 & 0.0083 & 0.0307 & 2 & 2.0204 & 0.0204 & 0.0349 \\
\hline
\end{tabular}




\section{Applications}

In this section, real data modeling performance of GIKw-W distribution is compared with several well-known distributions. We provide four applications to real data sets to illustrate the applicability of the GIKw-G family. We focus on the GIKw-W distribution introduced in Section 2. We compute the log-likelihood function evaluated at the MLEs $(\hat{\ell})$ using a limited-memory quasi-Newton code for bound-constrained optimization (L-BFGS-B). The goodness-of-fit measures, Anderson-Darling $\left(A^{*}\right)$, Cramèr-von Mises $\left(W^{*}\right)$ are computed for all models and used to decide the best model.The lower values of these statistics and higher p-values of K-S indicate good fits. The value for the Kolmogorov Smirnov (KS) statistic and its p-value are also provided. We compare the GIKw-W distribution with those of the Weibull (W), generalized inverted Kumaraswamy (GIKw) and Kumaraswamy (IKw).

The first data is corresponding to the vinyl chloride data obtained from clean upgrading, monitoring wells in $\mathrm{mg} / \mathrm{L}$ (Bhaumik et al., 2009). The data set are given as follows: 5.1, 1.2, 1.3, 0.6, $0.5,2.4,0.5,1.1,8.0,0.8,0.4,0.6,0.9,0.4,2.0,0.5,5.3,3.2,2.7,2.9,2.5,2.3,1.0,0.2,0.1,0.1,1.8$, $0.9,2.0,4.0,6.8,1.2,0.4,0.2$.

The second data set is obtained from Murthy et al. (2004). The data refers to the time between failures for repairable items. The data set are given as follows: $1.43,0.11,0.71,0.77,2.63,1.49$, $3.46,2.46,0.59,0.74,1.23,0.94,4.36,0.40,1.74,4.73,2.23,0.45,0.70,1.06,1.46,0.30,1.82,2.37$, $0.63,1.23,1.24,1.97,1.86,1.17$

The third data set can be obtained from Hinkley (1977) and it represents thirty successive values of March precipitation (in inches) in Minneapolis/St Paul. The data set are given as follows: $0.77,1.74,0.81,1.20,1.95,1.20,0.47,1.43,3.37,2.20,3.00,3.09,1.51,2.10,0.52,1.62,1.31,0.32$, $0.59,0.81,2.81,1.87,1.18,1.35,4.75,2.48,0.96,1.89,0.90,2.05$.

The fourth data is based on the waiting times between 65 consecutive eruptions of Blowhole (1998). The data set are given as follows: $83,51,87,60,28,95,8,27,15,10,18,16,29,54,91,8$, $17,55,10,35,47,77,36,17,21,36,18,40,10,7,34,27,28,56,8,25,68,146,89,18,73,69,9,37$, $10,82,29,8,60,61,61,18,169,25,8,26,11,83,11,42,17,14,9,12$.

The MLEs and the goodness of fit statistics of the models for all data sets are presented in Tables 2 to 9, respectively. As seen in Table 3, 5, 7, and 9 the GIKw-W model gives the lowest values. Therefore, it is clear that GIKw-W distribution provides the overall best fit and therefore could be chosen as the more adequate model for explaining data sets.

The histogram of the all data sets and the estimated pdf's and cdf's of the GIKw-W model and the other competitive models are displayed in Figures 5 and 6 . It is clear from Figures 5 and 6 that the GIKw-W model provide better fits to these four data sets. 
Table 2: The MLEs and their standard errors (in parentheses) for the first data set.

\begin{tabular}{cccccc}
\hline Distribution & $\alpha$ & $\beta$ & $\gamma$ & $\mathrm{a}$ & $\mathrm{b}$ \\
\hline GIKw-W & 3.8371 & 9.2165 & 0.1699 & 0.0120 & 1.5582 \\
& $(6.2626)$ & $(25.2396)$ & $(0.4264)$ & $(0.0279)$ & $(1.3545)$ \\
GIKw & 2.0265 & 0.8848 & 2.64300 & - & - \\
& $(1.8893)$ & $(0.6646)$ & $(3.8870)$ & - & - \\
IKw & 1.7408 & 2.1058 & - & - & - \\
& $(0.3237)$ & $(0.5373)$ & - & - & - \\
W & - & - & - & 0.5262 & 1.0102 \\
& - & - & - & $(0.1176)$ & $(0.1326)$ \\
\hline
\end{tabular}

Table 3: The goodness-of-fit statistics for the first data set

\begin{tabular}{lccccc}
\hline Distribution & $\mathrm{A}^{*}$ & $\mathrm{~W}^{*}$ & $\hat{\ell}$ & $\mathrm{K}-\mathrm{S}$ & $\mathrm{P}$-value \\
\hline GIKw-W & 0.1832 & 0.0270 & 54.7047 & 0.0770 & 0.9899 \\
GIKw & 0.2805 & 0.0379 & 55.7572 & 0.0955 & 0.9153 \\
IKw & 0.2764 & 0.0378 & 55.7706 & 0.0965 & 0.9090 \\
Weibull & 0.3000 & 0.0462 & 55.4496 & 0.0918 & 0.9366 \\
\hline
\end{tabular}

Table 4: The MLEs and their standard errors (in parentheses) for the second data set.

\begin{tabular}{cccccc}
\hline Distribution & $\alpha$ & $\beta$ & $\gamma$ & $\mathrm{a}$ & $\mathrm{b}$ \\
\hline GIKw-W & 0.1199 & 1.1017 & 3.3626 & 6.0015 & 1.1272 \\
& $(0.0277)$ & $(0.2661)$ & $(0.0524)$ & $(0.0066)$ & $(0.0066)$ \\
GIKw & 1.1631 & 1.847 & 1.4473 & - & - \\
& $(1.022)$ & $(1.1798)$ & $(1.3924)$ & - & - \\
\multirow{2}{*}{ IKw } & 2.4601 & 4.1715 & - & - & - \\
& $(0.4213)$ & $(1.2783)$ & - & - & - \\
W & - & - & - & 0.4560 & 1.4633 \\
& - & - & - & $(0.1141)$ & $(0.2029)$ \\
\hline
\end{tabular}

Table 5: The goodness-of-fit statistics for the second data set

\begin{tabular}{lccccc}
\hline Distribution & $\mathrm{A}^{*}$ & $\mathrm{~W}^{*}$ & $\hat{\ell}$ & $\mathrm{K}-\mathrm{S}$ & $\mathrm{P}$-value \\
\hline GIKw-W & 0.1813 & 0.0231 & 39.1069 & 0.0748 & 0.9989 \\
GIKw & 0.2653 & 0.0409 & 40.8189 & 0.1092 & 0.8665 \\
IKw & 0.3323 & 0.04948 & 41.2380 & 0.1111 & 0.8523 \\
Weibull & 0.1921 & 0.0299 & 39.9909 & 0.0788 & 0.9900 \\
\hline
\end{tabular}


Table 6: The MLEs and their standard errors (in parentheses) for the third data set.

\begin{tabular}{cccccc}
\hline Distribution & $\alpha$ & $\beta$ & $\gamma$ & $\mathrm{a}$ & $\mathrm{b}$ \\
\hline GIKw-W & 0.1308 & 1.2393 & 2.7063 & 4.7484 & 1.2909 \\
& $(0.0297)$ & $(0.3783)$ & $(1.6289)$ & $(0.0038)$ & $(0.0037)$ \\
GIKw & 1.9540 & 1.4209 & 3.9458 & - & - \\
& $(1.8055)$ & $(1.0186)$ & $(5.6701)$ & - & - \\
\multirow{2}{*}{$\mathrm{IKw}$} & 2.9876 & 8.5933 & - & - & - \\
& $(0.4731)$ & $(3.1240)$ & - & - & - \\
$\mathrm{W}$ & - & - & - & 0.3154 & 1.8088 \\
& - & - & - & $(0.0906)$ & $(0.2491)$ \\
\hline
\end{tabular}

Table 7: The goodness-of-fit statistics for the third data set

\begin{tabular}{lccccc}
\hline Distribution & $\mathrm{A}^{*}$ & $\mathrm{~W}^{*}$ & $\hat{\ell}$ & $\mathrm{K}-\mathrm{S}$ & $\mathrm{P}$-value \\
\hline GIKw-W & 0.1038 & 0.0144 & 37.9810 & 0.0547 & 0.9988 \\
GIKw & 0.3226 & 0.0514 & 30.3017 & 0.1097 & 0.8627 \\
IKw & 0.3505 & 0.0561 & 39.4255 & 0.1143 & 0.8280 \\
Weibull & 0.1693 & 0.0219 & 38.6432 & .0689 & 0.9901 \\
\hline
\end{tabular}

Table 8: The MLEs and their standard errors (in parentheses) for the fourth data set.

\begin{tabular}{cccccc}
\hline Distribution & $\alpha$ & $\beta$ & $\gamma$ & $\mathrm{a}$ & $\mathrm{b}$ \\
\hline GIKw-W & 0.1705 & 4.5176 & 5.4997 & 1.9419 & 0.5709 \\
& $(0.0212)$ & $(1.0214)$ & $(0.0069)$ & $(0.0029)$ & $(0.0028)$ \\
GIKw & 0.3096 & 4.2274 & 45.4405 & - & - \\
& $(1.1821)$ & $(16.1314)$ & $(16.8344)$ & - & - \\
IKw & 1.3768 & 60.9128 & - & - & - \\
& $(0.1377)$ & $(23.9816)$ & - & - & - \\
W & - & - & - & 0.0085 & 1.2685 \\
& - & - & - & $(0.0039)$ & $(0.1089)$ \\
\hline
\end{tabular}

Table 9: The goodness-of-fit statistics for the fourth data set

\begin{tabular}{lccccc}
\hline Distribution & $\mathrm{A}^{*}$ & $\mathrm{~W}^{*}$ & $\ell$ & $\mathrm{K}-\mathrm{S}$ & P-value \\
\hline GIKw-W & 0.7649 & 0.1039 & 293.2774 & 0.0981 & 0.6691 \\
GIKw & 1.2613 & 0.1848 & 295.4949 & 0.1018 & 0.5197 \\
IKw & 1.2044 & 0.1743 & 295.4044 & 0.0954 & 0.5047 \\
Weibull & 1.0078 & 0.1470 & 296.9085 & 0.1060 & 0.4681 \\
\hline
\end{tabular}


(a)

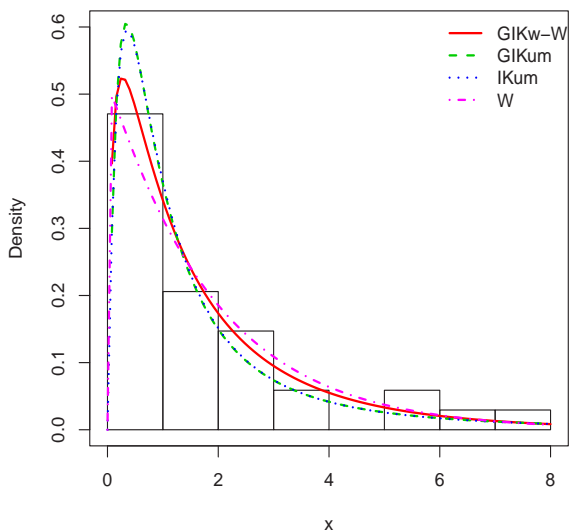

(c)

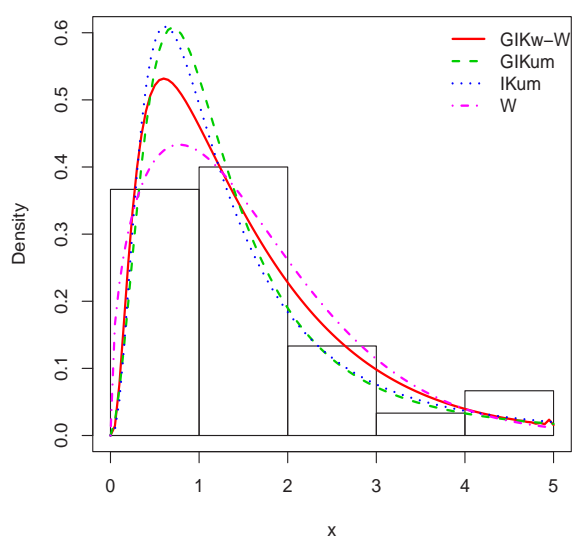

(b)

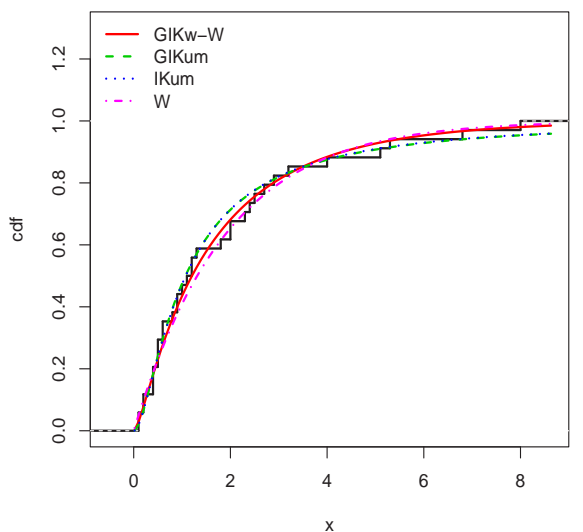

(d)

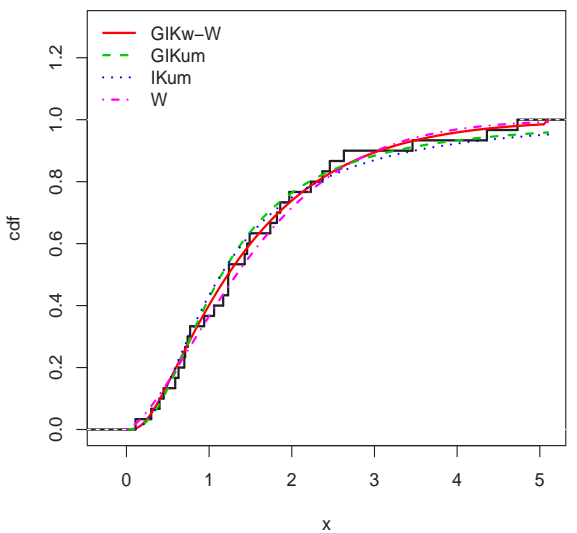

Figure 5: Estimated pdf in (a) and cdf in (b) for the first data set and estimated pdf in (c) and cdf in (d) for the second data set, respectively. 
(a)

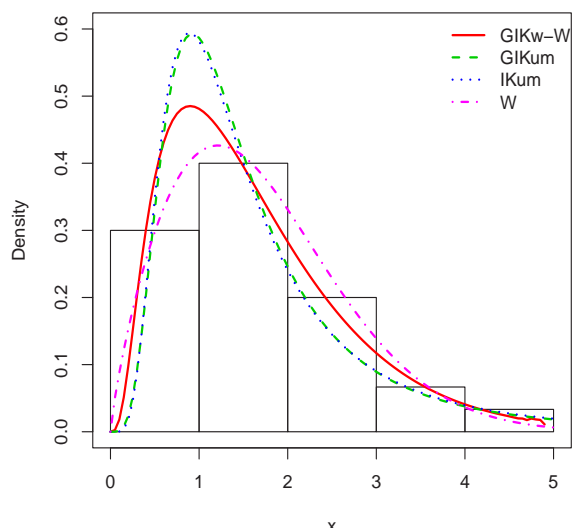

(c)

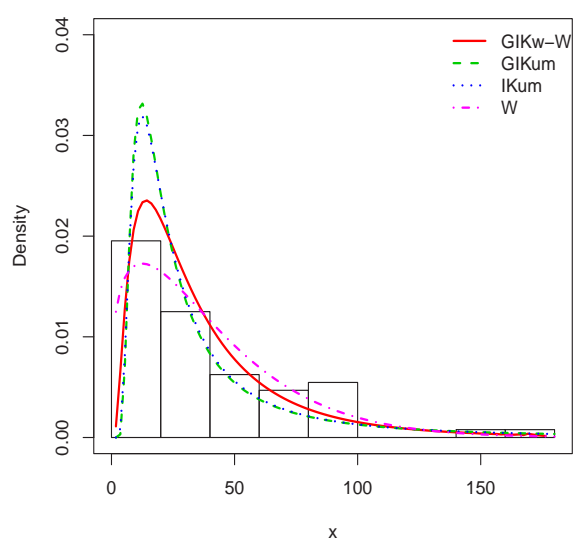

(b)

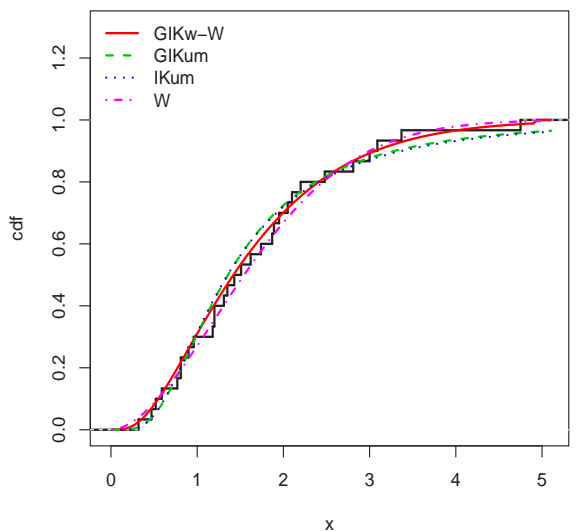

(d)

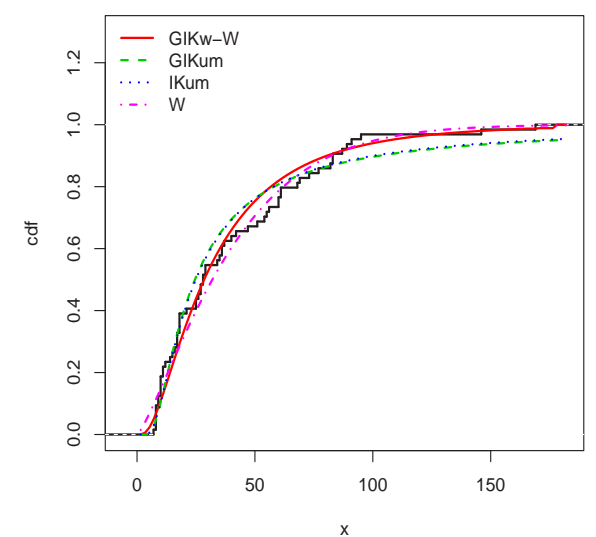

Figure 6: Estimated pdf in (a) and cdf in (b) for the third data set and estimated pdf in (c) and cdf in (d) for the fourth data set, respectively. 


\section{Conclusion}

This paper introduces a new class of models called the "GIKw-G" family of distributions which can generate all classical continuous distributions while is proved suitable for data of heavy-tails and exhibiting different features of skewness and reverse J-shapes. For any parent continuous distribution G, we define corresponding GKIw- G distribution. We study some of statistical and mathematical properties of the new generator, such as ordinary moments, quantile functions, Shannon and Reǹyi, entropies and order statistics. We discuss maximum likelihood estimates of the model parameters. Four applications of the new family and a simulation study demonstrate its usefulness and potentiality to analysis of real data. This new family and its generated models is commendably useful in several areas such as engineering, survival and lifetime data, hydrology, economics, among others.

\section{References}

[1] Abd El-Kader, R.I., AL-Dayian, G.R. and AL-Gendy, S.A. (2003). Inverted Pareto Type I distribution: properties and estimation. Journal of Faculty of Commerce AL-Azhar University, 21, 19-40.

[2] Al-Dayian, G.R. (2004). Inverted Pareto Type II distribution: Properties and estimation. Journal of Faculty of Commerce AL-Azhar University, 22, 1-18.

[3] Al-Dayian, G.R. (1999). Burr Type III distribution: Properties and estimation. The Egyptian Statistical Journal, 43, 102-116.

[4] Al-Fattah, A.M.A., EL-Helbawy, A.A. and AL-Dayian, G.R. (2017). Inverted Kumaraswamy Distribution: Properties and Estimation, Pakistan Journal of Statisitcs and Operation Research, 33(1), 37-67.

[5] Alexander, C., Cordeiro, G.M., Ortega, E.M.M., and Sarabia, J.M. (2012). Generalized beta generated distributions. Computational Statistics and Data Analysis, 56, 1880-1897.

[6] Aljuaid, A. (2013). Estimating the parameters of an exponentiated inverted Weibull distribution under Type II censoring. Applied Mathematical Sciences, 7(35), 1721-1736.

[7] Alzaatreh, A., Lee, C. and Famoye, F. (2013). A new method for generating families of continuous distributions. Metron, 71, 63-79.

[8] Arslan M. N., Tahir M. H., Jamal F., Ozel G.(2017). A New Generalized Burr Family of Distributions for the Lifetime Data, Journal of Statistics Applications \& Probability, 6, 1-17.

[9] Bhaumik, D.K., Kapur, K. and Gibbons, R.D. (2009). Testing parameters of a gamma distribution for small samples. Technometrics, 51, 326-334.

[10] Bourguignon, M., Silva, R.B., and Cordeiro, G.M. (2014). The Weibull-G family of probability distributions. Journal of Data Science, 12, 53-68.

[11] Kiama Blowhole Eruption. (1998). Retrieved from http://www.statsci.org/data/oz/kiama.html.

[12] Calabria, R. and Pulcini, G. (1990). On the maximum likelihood and least squares estimation in the inverse Weibull distribution. Journal of Statistica Applicate, 2(1), 53-66.

[13] Cordeiro G.M. and de Castro M. (2011). A new family of generalized distributions. Journal of Statistical Computation and Simulation, 81, 883-893.

[14] Cordeiro G.M., Ortega E.M.M., da Cunha D.C.C. (2013). The exponentiated generalized class of distribution. Journal of Data Science, 11, 1-27. 
[15] Cordeiro, G.M., Ortega, E.M.M., Bozidar, P.V., Pescim, R.R. (2014). The Lomax generator of distributions: Properties, minification process and regression model. Applied Mathematics and Computation, 247, 465-486.

[16] Elgarhy, M., Hassan, A.S. and Rashed, M. (2016). Garhy - Generated Family of Distributions with Application, Mathematical Theory and Modeling, 6, 1-15.

[17] Elgarhy, M., Haq, M.A., Ozel, G. and Nasir, A. (2017). A New Exponentiated Extended Family of Distributions with Applications, Gazi University Journal of Sceince, 30(3),101-115.

[18] Eugene, N., Lee C., and Famoye, F. (2002). Beta-normal distribution and its applications. Communication in Statistics - Theory Methods, 31, 497-512.

[19] Hassan, A. S. and Elgarhy, M. (2016a). Kumaraswamy Weibull-generated family of distributions with applications. Advances and Applications in Statistics, 48, 205-239.

[20] Hassan, A. S. and Elgarhy, M. (2016b). A new family of exponentiated Weibull-generated distributions. International Journal of Mathematics And its Applications, 4, 135-148.

[21] Hassan, A. S., Elgarhy, M., and Shakil, M. (2017). Type II half logistic family of distributions with applications. Pakistan Journal of Statistics and Operation Research, 13(2), 245-264.

[22] Hassan, A. S. and Hemeda, S. E. (2016). The additive Weibull-g family of probability distributions. International Journals of Mathematics and Its Applications, 4, 151-164.

[23] Hinkley, D. (1977). On quick choice of power transformations, Journal of the Royal Statistical Series C, 26(1), 67-69.

[24] Jones, M.C. Families of distributions arising from the distributions of order statistics. (2004). Test, 13, $1-43$.

[25] F. Jamal, M.A. Nasir, M.H. Tahir and N.H. Montazeri, The odd Burr-III family of distributions. Journal of Statistics Applications and Probability, 6, 105-122 (2017).

[26] Murthy, D.N.P., Xie, M. and Jiang, R. (2004). Weibull Models, Wiley Series in Probability and Statistics, John Wiley and Sons, New York.

[27] Prakash, G. (2012). Inverted exponential distribution under a Bayesian view point. Journal of Modern Applied Statistical Methods, 11(1), 190-202.

[28] Reǹyi, A. On measures of entropy and information. (1961). In: Proceedings of the 4th Fourth Berkeley Symposium on Mathematical Statistics and Probability, University of California Press, Berkeley, 547561.

[29] Ristič, M.M., and Balakrishnan, N. (2012). The gamma-exponentiated exponential distribution. Journal of Statistical Computation and Simulation, 82, 1191-1206.

[30] Shannon, C.E. (1948). A mathematical theory of communication. Bell System Technical Journal, 27, 379-432.

[31] Tahir, M.H., Cordeiro, G.M., Alzaatreh, A. Mansoor, M., Zubair, M.The logistic-X family of distributions and its applications, Communication in Statistics-Theory and Methods, 45(24), 7326-7349.

[32] Torabi H, Montazari NH. (2012). The gamma-uniform distribution and its application. Kybernetika. 48, 16-30.

[33] Torabi, H., Montazari, N.H. (2014). The logistic-uniform distribution and its application. Communications in Statistics: Simulation and Computation, 43, 2551-2569.

[34] Zografos, K. and Balakrishnan, N. (2009). On families of beta- and generalized gamma-generated distributions and associated inference. Statistical Methodology, 6, 344-362. 